\title{
Silicone rubber in defects of the conjunctiva
}

\author{
M. MERZ AND W. GZERWIŃSKA \\ From the Department of Ophthalmology, WAM School of Medicine, Warsaw, and the Department \\ of Ophthalmology, Medical Academy, Warsaw, Poland
}

The material commonly used today for filling up conjunctival defects is obtained from the buccal mucosa or the skin. The taking of these grafts has some drawbacks, such aif scarring and tenderness of the areas from which the flaps have to be excised. Skin grafts into the conjunctival sac have also many well-known cosmetic disadvantages. The use of silicone rubber has many advantages, such as good tolerance by living tissue, adaptability and smoothness. Silicone rubber does not interfere with the exchange of gases, and i allows a normal metabolism in the underlying tissues. Bowen and Dyer (1962) used silicone rubber grafts in muscle lengthening procedures in cases of squint. They observedg in experimental investigations that the implanted graft was quickly covered by a thin sheefe of connective tissue, with cells similar to endothelial cells on the surface adjacent to thछ silicone. Morales, Polack, and Arata (1966), in similar experiments, found good tolerances of this material in man, and showed by microscopical examinations that the surface of the silicone implant was covered by a thin sheet of connective tissue. Agnew, Todd Richmond, and Chronister (1962) pointed out that silicone rubber implanted into the peritoneum, subcutaneous tissue, and brain of experimental animals provoked only ఖ slight tissue reaction and was encapsulated by connective tissue, which did not adhere t氶 the implant. The formation of a connective tissue capsule around the implant is also confirmed by the observations of Leininger, Mirkovitch, Peters, and Hawke (1964). If comparison with the capsule formed after the implantation of Teflon, nylon, malar, and polyethylene, the capsule in cases of silicone implant is thinner and the tissue reaction the least.

The case described below shows how a silicone rubber graft may be used in large posto operative defects of the conjunctiva.

\section{Case report}

A 29-year-old male was referred to the Ophthalmic Department in I 966 for enucleation of the lef( eye. For the previous 3 years this eye had been blind, but the patient had been unwilling to have i⿺ removed. In 1964 he had observed a small, dark nodule situated in the upper temporal quadran of the left eyeball; it was enlarging quickly so that 2 years later it appeared in the palpebral fissure오 The patient was not severely ill, and the family history did not reveal any relevant details.

Examination His general health was good. The right eye was normal with visual acuity 6/6.

The left lids were normal, but in the palpebral fissure there was a greyish-brown tumour the siz衰 of a cherry, of uneven, cauliflower-like, surface covered by a fibrous capsule. It was heavily vas? cularized. A thick, short peduncle connected it with the sclera in the region of the ciliary body aq 2 o'clock. Small clumps of pigment were scattered in the hyperaemic conjunctiva close to the 
tumour (Fig. I); the upper and temporal part of the cornea was covered by the tumour, and through the remaining transparent part of the cornea a greyish-brown, partly atrophic iris and a cataractous lens could be seen (Fig. 2). The eye was very hard and blind.

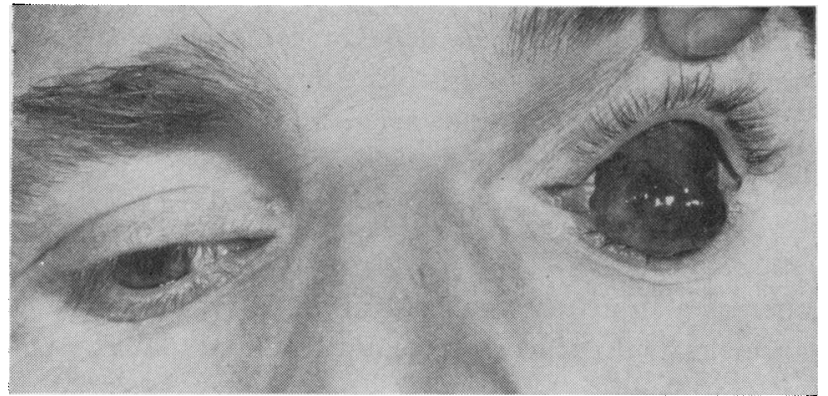

FI G. I Front view of the patient's eyes when looking down

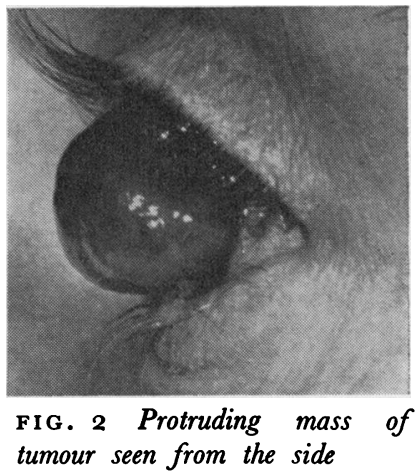

tumour seen from the side

Laboratory findings did not reveal any abnormalities. A scintigram of the liver showed normal tissue. The eye had to be removed either by enucleation or by orbital evisceration, but the patient would not consent to the latter.

Surgery The chief surgical problem was to replace a vast area of the bulbar conjunctiva, since the whole upper and temporal sectors extending up into the fornix had to be excised.

An oval flap of silicone rubber, 20 by $15 \mathrm{~mm}$. approx. was fixed by four plain catgut mattress sutures near the fornix at the beginning of the procedure. After the enucleation had been completed six more mattress sutures were added, fixing the lower and medial conjunctival wound lips so that the border of the silicone flap was kept underneath the conjunctiva. A plastic ball was inserted into the conjunctival sac and a pressure dressing maintained for 3 days.

Result Postoperative oedema of the lids persisted for nearly a week. One week after surgery the borders of the silicone flap were in full apposition with the neighbouring conjunctiva, and in following days a well-vascularized conjunctiva began to encroach on the flap. Four weeks after surgery the implant was already covered by a smooth, well-vascularized conjunctival sheath, except for a small area below. Three months after surgery the protruding corner of the silicone flap was grasped by forceps and slipped out. After 18 months follow-up there was nothing abnormal in the appearance and behaviour of the conjunctiva in comparison with a normal one as seen after enucleation in other patients (Fig. 3).

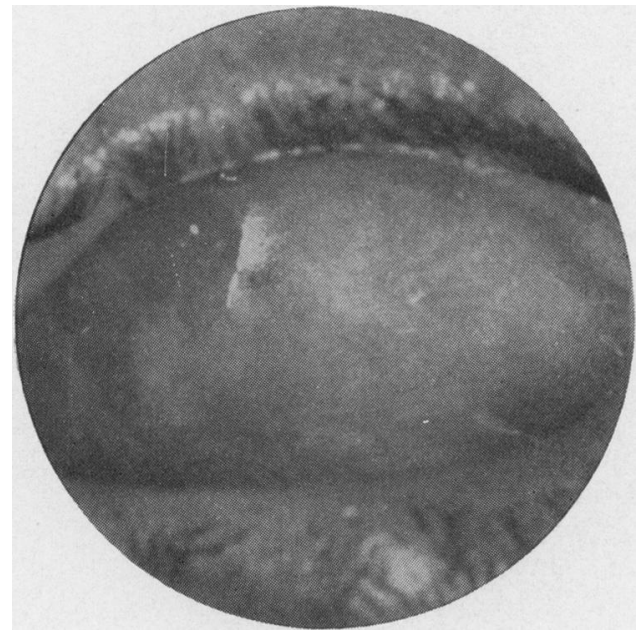

FIG. 3 Conjunctival sac 3 months after surgery 


\section{Discussion}

Experimental and clinical investigations point to a steadily increasing role of silicone materials in ophthalmology. In the case presented a silicone rubber graft was used as $\frac{0}{8}$ substitute material and as a framework for the mucosa to grow over. It was then easil removed after the new tissue had filled up the defect. The silicone rubber is so smoot that the connective tissue fibres cannot grow into it, but form a capsule over it coverin 8 the defect with new tissue. The cosmetic results are nearly perfect, and much better that those obtained with a graft from the buccal mucosa.

\section{Summary}

The use of silicone rubber as a substitute material in an extensive conjunctival defect described. New tissue grew over the silicone implant which was later removed leaving \& normal conjunctiva.

\section{References}

AGNEW, W., TODD, E., RIGHMOND, H., and CHRONISTER, W. s. (1962) $\quad$ F. surg. Res., 2, 357

BOWEN, S. F., jr., and DYER, J. A. (1962) Invest. Ophthal., r, 579

LeININGER, R., MIRKovitch, v., PETERS, A., and haWke, w. (1964) Bull. Dow Corning Center, 6, No. 9

p. 10

MORAles, A., POlACK, F. M., and ARAtA, A. F. (1966) Brit. J. Ophthal., 50, 235 